\title{
New Anti-Angiogenic Targeted Therapy in Advanced Renal Cell Carcinoma (RCC). Current Status and Future Prospects
}

\author{
Alessandro Sciarra*, Vincenzo Gentile, Stefano Salciccia, Andrea Alfarone and Franco Di Silverio \\ Resident in Urology, Department U Bracci, University Sapienza, Rome, Italy
}

\begin{abstract}
:
Objectives: To address the rationale for anti-angiogenic targeted therapies in advanced RCC.

Methods: We reviewed the international recent literature ,using Pubmed search.

Results: RCC is genetically linked to factors regulating angiogenesis, in particular vascular endothelial growth factor (VEGF). Sunitinib is a multitarget receptor tyrosine-kinase (TK) inhibitor, acting on VEGF receptor (VEGFR) and platelet-derived growth factor receptors (PDGFR). Sorafenib is an oral multikinase inhibitor (VEGFR and PDGFR) showing also inhibitors effect on the Raf system.

Phase I trials showed no life threatening toxicities relates to these agents. Phase II and phase III trials showed that these antiangiogenic agents are effective in the treatment of advanced RCC, mainly in cytokine refractory metastatic RCC. Survival benefits exist in particular when advanced RCC patients undergo cytoreductive nephrectomies before the initiation of the systemic therapy.

To better use this kind of targeted therapy in advanced RCC, different points must be developed: the identification of clinical characteristic of RCC able to predict outcomes and responses to therapy; differences among different compounds; advantages of combination or sequential therapies.

Conlusions: Targeted therapy with Sunitinib and Sorafenib has been approved to FDA and is revolutioning how we clinically approach advanced RCC.
\end{abstract}

Key Words: Renal cell carcinoma, kidney neoplasm, tyrosin kinase, medical therapy.

\section{INTRODUCTION}

The incidence of renal cell carcinoma (RCC) has been increasing over the past decades. In the US, more than 38000 new cases and 12000 deaths were estimated to occur from RCC in 2006 [1]. In 2007, it is evaluated that approximately 51000 people will be diagnosed and 12000 deaths will be related to cancers of the kidney (the vast majority are RCC) in US [2]. The diagnostic trend is mainly due to the use of non-invasive abdominal imaging procedures, which show incidental renal tumors. The majority of incidentally detected RCC are at low stage. The management of clinically localized RCC is represented by surgical extirpation and it represents a consistent approach to offer a cure for patients with this localized disease. However, a stable proportion of $20 \%-30 \%$ of patients still present with metastatic disease and $20 \%-30 \%$ of cases who undergo curative surgery will develop metastatic disease [3]. Treatment options for metastatic RCC have been in the past limited because of tumor resistance to chemotherapy and radiotherapy. The lack of demonstrable efficacy of chemotherapies and radiotherapies in advanced RCC has lead to a 5-years survival ranging from 5\% to $10 \%[4]$.

The immunogenity of RCC has represented the basis supporting the use of immunotherapy in advanced RCC. As immunotherapeutic agents either interleukin-2 (IL-2) and interferon (IF) have been used. High dose IL-2 was approved by US FDA, as it leads to occasional durable complete responses [5]. However, due to its toxicity, a minority of patients are eligible for high-dose IL-2 therapy and only $5 \%$ experience a durable complete remission [5]. IF therapy leads to modest improvements in survival [6]. Combination of IL-2 and IF has not significantly improved clinical results [7]. For these reasons, new therapies for metastatic RCC patients have long been sought. In the last years, new advances have begun to revolutionize the management of advanced RCC and offer hope for the future. First, a proven role for aggressive surgical resection of the primary lesion and metastatectomy has been underlined. Second, a series of exciting new approaches ,so called "Targeted therapy", are revolu-

*Address correspondence to these authors at the Department Urology U Bracci, University Sapienza, Viale Policlinico 155, 00161 Rome, Italy; Tel/Fax: +39-06-4461959; E-mail: sciarrajr@hotmail.com tioning the management of advanced RCC. In particular, recent progress in understanding the biology of RCC has led to the identification of angiogenesis and vascular endothelial growth factor (VEGF) as a significant therapeutic target in patients with advanced RCC [8]. Several studies using strategies to inhibit VEGF activity have demonstrated significant anti-tumor effects in the management of RCC. Differences in histological patterns of RCC seem to influence the response to new targeted therapies, and most data are documented in clear cell than in papillary or chromofobe histology. The present review will address in particular the experimental rationale for this targeted therapy, the mechanism of action of drugs proposed, results from clinical trials, strategies to maximize the potential of these agents in advanced RCC. We will try to highlight how far we have came and how far we still to go.

\section{METHODS FOR THE REVIEW}

Several reviews related to this topic have been published; here we will focus on new findings and suggestions. In particular we reviewed the international recent literature using PUBMED search (advanced RCC, targeted therapy), so to analyze new findings on the role of anti-angiogenic / VEGF targeted therapies in advanced RCC.

\section{EXPERIMENTAL RATIONALE FOR TARGETED THER-} APY

\section{Angiogenesis}

The approach of targeting the renal cancer cell itself has thus far been largely unsuccessful.

The clinical knowledge that RCC is a highly vascular cancer and that the Von Hippel Lindau (VHL) protein has an important role in sporadic RCC, have made anti-angiogenic strategies an attractive approach [9]. RCC is genetically linked to factors regulating angiogenesis, such as VEGF, platelet-derived growth factor(PDGF) and basic fibroblast growth factor(bFGF) $[8,10,11]$. VEGF is the strongest pro-angiogenic protein in RCC and there is a strong rationale for blocking VEGF in this tumor. Approximately $60 \%$ of clear RCC have an inactivated VHL tumor suppressor gene (50\% through somatic mutation and $10 \%$ through promoter methy- 
lation). A normal VHL protein indirectly blocks transcription of hypoxic-inducible genes, such as VEGF $[12,13]$. Therefore, under hypoxic conditions or VHL gene inactivation, there is an induction of VEGF transcription, over-expression of VEGF protein and angiogenesis [12,13]. In addition to its angiogenic properties VEGF may also suppress anti-tumor immune responses by inhibiting recruitment and activation of dendritic cells [14]. Different studies showed that nearly all RCC express high levels of VEGF and that this over-expression correlates with tumor progression and prognosis [15]. VEGF has several isoforms: VEGF-A is involved in angiogenesis, whereas VEGF-C and VEGF-D are more related to lymphoangiogenesis [16,17]. Leppert et al. [18] immunoistochimically analyzed the expression of VEGF-A, VEGF-C, VEGF-D, VEGF-1, VEGF-2 and VEGF-3 in RCC. The mean expression percentage within the tumor epithelium was $37 \%, 71 \%, 51 \%, 54 \%, 49 \%$ and 13\%. Lam et al. [19] showed the predictive value for both presence of metastasis and disease-specific survival of these proteins of the VEGF family. In particular low endothelial expression of VEGF-3 was independently associated with both lymph-node metastasis and poor prognosis[19]. Leclercq et al. [20] analyzed in RCC the expression of VEGF in tumor cells, the relationship between VEGF expression and VEGF plasma levels and the association with usual clinical and pathological prognosis factors. The median plasma VEGF level was $129 \mathrm{pg} / \mathrm{ml}$; the median value of VEGF staining was $50 \%$. All RCC cells exhibited a weak diffuse and membranous VEGF staining and $42 \%$ cases presented strong intra-cytoplasmic and membranous VEGF signal. They also found that VEGF expression was increased at the rims of necrotic tumor areas [20]. Tissue VEGF expression significantly correlated with plasma VEGF level, the presence of tumor necrosis, nuclear Fuhrman grade, tumor stage and progression [20]. Plasma VEGF levels significantly correlated with Fuhrman grade, tumor stage and the presence of lymph-node or distant metastases [20]. In addition, RCC with tumor necrosis has a median VEGF expression of 80\%, plasma VEGF level of 187 $\mathrm{pg} / \mathrm{ml}$ compared with RCC with no tumor necrosis that had a median VEGF expression of 20\% and plasma VEGF level of 104 $\mathrm{pg} / \mathrm{ml}$.

VEGF represents a significant mediator for tumor growth and metestasis development via specific receptor tyrosine kinases that are up-regulated during angiogenesis [21]. Moreover VEGF plays major roles in different steps of tumor development with increase of microvascular permeability, permeabilization of blood vessels, extravasation of plasma proteins, introduction of endothelial cell division and migration, promotion of endothelial cell survival [20]. Hypoxia in RCC plays an important role in the VEGF pathway. When tumors are exposed to hypoxia as a result of a rapid growth beyond existing blood supplies, the tumor become necrotic. Larger tumors have an inadequate blood supply and thus, hypoxia occurs, causing up-regulation of VEFG [20]. For this reason in the perinecrotic areas the hypoxic conditions are a major stimulus for the production of VEGF. The correlation between serum levels of VEGF and prognostic parameters remains controversial. Jacobsen et al. [22] found a difference between plasma VEGF levels in patients with RCC and controls and showed that plasma VEGF levels were correlated with tumor volume. On the contrary Edgren et al. [23] found no correlation between plasma VEGF levels and clinical or pathological markers or patient survival in RCC.

There are two possible approaches for targeting VEGF signaling : ligand blockade through a monoclonal antibody (Bevacizumab), and blocking signalling by targeting the receptors with either a monoclonal antibody or a small-molecule tyrosine kinase inhibitor (Sorafenib and Sunitinib).

\section{Tyrosine Kinase Receptors (TKR)}

TKR are transmebrane proteins at the cell surface that transduce extracellular signals to the cytoplasm [24]. These proteins contain extracellular ligand-binding domains and intracellular catalytic domains [24]. Ligand binding induces dimerization of these TKR and activation of tyrosine kinase (TK). TK activity stimulates multiple signalling pathways that modulate DNA syntesis, cell division, growth, progression, differentiation, migration [24]. VEGF, PDGF, bFGF, receptors posses TK activity that promotes intracellular signalling [25].

\section{Ras/Raf and Epidermal Growth Factor Receptors (EGFR) Pathways}

Another important pathways in RCC is the Ras/Raf pathway. This signalling starts by binding of a ligand to 1 of the 4 erB proteins, the most prominent is erbB protein which is also called the EGFR [11]. The EGFR consists of 3 domains; a ligand binding domain, a transmembrane domain and a cytoplasmic domain with TK activity. After binding of the ligand (EGF), the EGFR is activated and it subsequently allows the Ras protein to be activated. The Ras protein then binds to Raf kinase and activates its function. This process finally, leads to regulation of transcription of important proteins such as S6 kinase [11]. Activation of Ras signalling pathways is an important mechanism by which human cancer develop. Activation of Ras occurs through mutational activation of the Ras oncogene or it can also be facilited by overexpression of a variety of TKs. In this way, most human tumors (including RCC), depend upon activation of the Ras signal transduction pathways to achieve cellular proliferation and progression.

Targeting EGFR pathways might also be an attractive intervention. The fact that EGFR is expressed in $50-90 \%$ of RCC makes appealing the possibility that this pathways may represent a potential anti-tumor target [26]. There are two approaches to the inhibition of EGFR: small molecule inhibitors of the intracellular TK, and monoclonal antibodies that target the extracellular domain. However studies with small molecules such as Gefitib (Iressa) or antibodies against EGFR, Cetuximab and Abfenix, have shown limited antitumor effect in RCC $[27,28]$.

\section{mTOR Pathway}

The mammalian target of rapamycin (mTOR) pathway has a central role in the regulation of cell growth, and it appears to be dysregulated in cancer [11]. mTOR receives stimuli from growth factors, hormones, nutrients and it stimulates proteins syntesis. The mTOR pathway also contributes to angiogenesis [11]. A UCLA study showed that mTOR pathways is affected in RCC patients with poor prognostic factors [29]. As inhibitors of this pathway, at present there are 3 mTOR inhibitors that are in clinical development as anticancer agents: Temsirolimus, AP23572 and RAD001.

\section{MET Proto-Oncogene and Nuclear Factor -KB}

The MET proto-oncogene encodes a transmembrane receptor with TK activity. Mutation of the MET proto-oncogene was frequently observed in hereditary papillary RCC but also in a subset of the sporadic tumors [30]. Moreover, trisomy of chromosome 7, the chromosome that contains the MET gene, is the most frequent aberration in sporadic papillary RCC [11]. MET protein expression has been observed either in papillary or clear cell RCC [30], and was reported as a significant indicator for survival among these patients.

Another strategy in particular for non clear cell RCC might be to target nuclear factor-KB (NF-KB). NF-KB interacts as a transcription factor with genes that cause production of proteins and that are essential for cell growth. Bortezomib represents a NF-KB pathway inhibitor, under clinical evaluation [11].

\section{Carbonic Anhydrase IX}

Carbonic anhydrase IX (CAIX) may represent a significant marker in RCC [11,31]. CAIX is a transmembrane enzyme that plays a role in the adaptation of the tumor to hypoxic conditions. 
Table 1. Prognostic Variables in Advanced RCC. Risk Level Stratification by the University of California Integrated Staging System (UISS) (3)

\begin{tabular}{|c|c|c|c|c|c|}
\hline Prognostic Variable & \multicolumn{2}{|c|}{ Low Risk Level } & \multicolumn{2}{c|}{ Intermediate Risk Level } & High Risk Level \\
\hline \hline T Stage & N1 M0 & N2 M0 or M1 & N2 M0 or M1 & N2 M0 or M1 & M0 or M1 \\
\hline Fuhrmann grade & any & $1-2$ & $3-4$ & $1-3$ & 4 \\
\hline ECOG score & any & 0 & 0 & $\geq 1$ & $\geq 1$ \\
\hline
\end{tabular}

CAIX expression in normal tissue is limited to the gastrointestinal tract, gold bladder and pancreas whereas over-expression of CAIX has been seen in many tumors, including RCC [31]. Moreover CAIX is regulated through VHL [31]. In RCC, both hypoxia and loss of VHL function lead to up-regulation of CAIX. CAIX can be also used as a therapeutic target in 2 strategies. The first is to target CAIX using anti-CAIX antibody (WX-G250). The second is to use a vaccination stimulating the host immune system to generate CAIX targeting cytotoxic T lymphocytes [11,31].

\section{CLINICAL RATIONALE FOR A TARGETED THERAPY: THE CONCEPT OF CYTOREDUCTIVE SURGERY}

An important consideration in the management of advanced $\mathrm{RCC}$ is the ole of removing the primary tumor, the cytoreductive nephrectomy. Different retrospective studies on metastatic RCC cases identified nephrectomy as a favourable treatment [32]. Moreover, retrospective analysis showed that metestatic RCC cases treated with primary nephrectomy and cytockines had better results than cases without prior nephrectomy [32]. On the basis of these data , 2 prospective randomized trials were initiated on T-any, Nany, M1 RCC cases and ECOG performance status 0 or 1 . The Southwest Oncology Group (SWOG) randomized 241 metastatic RCC cases to IF- alone or nephrectomy followed by IF- $\alpha$ therapy [32]. Patients submitted to cytoreductive nephrectomy had a survival advantage over those receiving IF- $\alpha$ alone (11.1 versus 8.1 months respectively: $\mathrm{p}=0.012$ ) [33]. The second trial, the European Organization For Research and Treatment of Cancer (EORTC) trial randomized 85 advanced RCC patients to IF- $\alpha$ alone versus nephrectomy plus IF- $\alpha$ [34]. Also in this trial the median survival was significantly better for the surgery arm (17 versus 7 months: $\mathrm{p}=0.010)$. The benefit of cytoreductive surgery was more evident in ECOG score 0 cases, but not dependent on site of metastasis.

All these data strongly suggest that an overall survival benefit exists when advanced RCC patients undergo cytoreductive nephrectomy before the initiation of a systemic therapy. This concept has been proved using immunotherapy as a systemic therapy but it should be proved also for targeted therapy as systemic treatment. In this case, proper patient selection is essential to maximize the benefits of cytoreductive nephrectomy. Garcia et al. [35] considered optimal candidates those with (1) good performance status, (2) a resectable primary tumor representing the majority of tumor borden, (3) no evidence of central nervous system metastases, (4) no prohibitive medical conditions, (5) no evidence of rapidly progressive extra-renal disease.

Resection of solitary (or limited in number) metastases can be associated to significant results in $30 \%$ of such patients [35]. Good results have been obtained in cases with pulmonary metastases or also after excision of hepatic, adrenal, brain and pancreatic metastases [35].

At now there is an increasing interest in using integrated staging system to select RCC cases in different prognostic categories. TNM stage, Furhman grade and ECOG score, compose the University of California Integrated Staging System (UISS) which now stratifies 6 categories, 3 for non-metastatic and 3 for metastatic RCC [3] (Table
1). A second validated score is the SSIGN (stage, size, grade, necrosis) based on the Mayo Clinic experience on RCC cases [36]. These systems may be useful for the selection of high risk patients most suitable for adjuvant treatment trials and the assignment of patients with metastatic RCC to different treatment strategies and different targeted therapies.

\section{MECHANISM OF ACTION OF VEGF-TARGETED THERAPIES}

The main targeted therapy in RCC recently analysed is that against angiogenesis and VEGF signalling pathway. This targeted therapy appears to be associated with more favourable clinical results. In the present review, we limited the analysis of the mechanism of action and that of the results from clinical trials, to these specific anti-VEGF therapies.

\section{Bevacizumab}

Bevacizumab is a recombinant human antibody against VEGF binds and it neutralizes all biologically active isoforms of VEGF [37]. This mechanism leads to vascular regression, living the tumor dormant. In solid tumors Bevacizumab acts through 3 potential mechanisms: 1) repression of microvasculature, 2) normalization of mature vasculature, 3 ) inhibition of the production of new vasculature [38]. In RCC the direct effect of Bevacizumab on microvasculature, leads to tumor regression and slowing the tumor growth.

\section{Sunitinib}

Sunitinib is a recent small molecule, acting as multi-targeted receptor tyrosine kinase inhibitor. [35,38]. It showed anti-tumor activity against RCC cells dependent upon signalling through PDGFR, VEGFR, and stem cell factor receptor (KIT) for proliferation and progression [38]. Sunitinib therefore may display a direct antitumor effect through KIT and indirect anti-tumor effect through anti-angiogenic effect (PDGFR,VEGFR).

\section{Sorafenib}

Sorafenib is an oral multikinase inhibitor that was originally developed because of its inhibition on the Raf system and on several TKs that regulate cell proliferation and angiogenesis [39]. In particular Sorafenib targets the VEGF and PDGF family and the kinase activity of both C-Raf and B-Raf. Together these data suggest that Sorafenib in RCC may inhibit tumor growth by a duel mechanism of action: either directly on the tumor (Raf signalling) and/or indirectly on tumor angiogenesis (VEGFR and PDGFR signalling).

\section{CLINICAL RESULTS FROM PHASE I-III TRIALS}

Different phase I-III clinical trials using Bevacizumab, Sorafenib and Sunitinib have been developed and all show that each of these anti-angiogenic agents is effective in the treatment of metastatic RCC (Table 2). In most of these studies, the population analyzed was represented by metastatic RCC in progression after cycles of cytokine therapies. 
Table 2. Selected Trials on VEGF - Targeted Agents in Advanced RCC

\begin{tabular}{|c|c|c|c|c|c|c|c|}
\hline Agent & Target & No. of Cases & Trial & Prior Therapies & ORR (\%) & PFS (Months) & $\begin{array}{c}\text { Overall Sur- } \\
\text { vival (months) }\end{array}$ \\
\hline Bevacizumab (39) & VEGF & 116 & Phase II versus placebo & Cytokines & 10 versus 0 & 4.8 versus 2.5 & NA \\
\hline Bevacizumab (40) & VEGF & 150 & $\begin{array}{c}\text { Phase II versus Bevacizu- } \\
\text { mab + Erlotinib }\end{array}$ & None & 13 versus 14 & 8.5 versus 9.9 & NA \\
\hline Sunitinib (44) & VEGFR & 63 & Phase II single agent & Cytokines & 40 & 8.7 & NA \\
\hline Sunitinib (45) & VEGFR & 106 & Phase II single agent & Cytokines & 44 & 8.1 & NA \\
\hline Sunitinib (46) & VEGFR & 750 & Phase II versus IF-a & None & 31 versus 6 & 11.0 versus 5.0 & NA \\
\hline Sorafenib (50) & VEGFR and Raf & 202 & Phase II versus placebo & Cytokines & NA & 5.5 versus 1.5 & NA \\
\hline Sorafenib (51) & VEGFR and Raf & 905 & Phase II versus placebo & Cytokines & 2 versus 0 & 5.5 versus 2.8 & 19.3 versus \\
& & & & & & \\
\hline
\end{tabular}

\section{Bevacizumab}

The clinical efficacy of Bevacizumab in metastatic RCC was first investigated in a randomized phase II trial in which 116 cases received placebo versus low-dose $(3 \mathrm{mg} / \mathrm{kg})$ Bevacizumab, versus high dose $(10 \mathrm{mg} / \mathrm{kg})$ Bavacizumab (intravenously every 2 weeks) [39]. All patients showed disease progression after at least one cycle of systemic cytokines treatment (95\% IL-2). No life-threatening toxicities or deaths related to Bevacizumab were reported. Toxicity episodes included hypertension (36\% of cases), proteinuria and epistaxis more commonly in the high dose Bevacizumab arm.

All toxicity episodes were reversible after cessation of the therapies. The study showed that time to progression was significantly longer in patients treated with high dose Bevacizumab (4.8 months) than in those with placebo (2.5 months; $\mathrm{p}=0.001$; $\mathrm{HR}=2.55$ ). No differences between placebo and low dose Bevacizumab arms in terms of progression-free survival (PFS) were found. In this trial the tumor burden reduction observed in Bevaciumab arms, did not meet criteria for objective response (Response Evaluation Criteria in Solid Tumors (RECIST) as $\geq 30 \%$ tumor burden reduction).

More recently the effect of Bevacizumab monotherapy in untreated RCC was defined in a randomized phase II trial of Bevacizumab $(10 \mathrm{mg} / \mathrm{kg}$ every 2 weeks) \pm Erlotinib (an oral EGFR receptor TK inhibitor, $150 \mathrm{mg} /$ day) [40]. The objective response rate (ORR) was $13 \%$ and median PFS with Bevacizumab monotherapy was 8.5 months. The addition of Erlotinib did not improve clinical results. Two different randomized phase III trials using Bevacizumab in combination with IF versus IF alone have completed accrual ( CALGB-90206 and B017705) [41].

\section{Sunitinib}

The recommended dose for Phase II trials using Sunitinib, was defined in phase I trials as $50 \mathrm{mg} /$ day orally for 4 weeks, followed by 2 weeks off, in repeated 6 weeks cycles [24,42]. A recent metaanalysis of the pharmacocynetic of Sunitinib from 10 phase I studies, determined that hepatic and renal function had no effect [43]. In phase I studies, fatigue was the most commonly reported adverse event (70\% of cases included) [25]. This symptom was generally associated with lethargy, asthenia, weakness. Hypothyroidism was also observed in approximately $65 \%$ of treated cases who underwent thyroid testing in clinical trials [24]. The results of phase I trials suggest that thyroid dysfunction should be considered in the differential diagnosis of fatigue during Sunitinib therapy. The most common grade of toxicities reported included fatigue $(10 \%)$, neutropenia (8\%), thrombocytopenia (7\%) [24].

Two phase II trials have been conducted in patients with metastatic RCC in progression after cytokine therapies [44,45]. In a population of 168 evaluable patients, Sunitinib induced an ORR of
$40 \%$ with a median time to progression of 8.7 months and a median survival of 16.4 months. A longer PFS was observed in patients with favourable ECOG score and normal serum haemoglobin. These results induced US Food and Drug Administration (FDA) approval of Sunitinib for the treatment of advanced RCC. Recently a phase III randomized trial of Sunitinib as first line therapy have been reported (Sunitinib versus IF in 750 metastatic RCC) [46]. The median PFS was 11 months for the Sunitinib arm and 5 month for the IF arm $(H R=0.415, p<0.0001)$. The ORR was $31 \%$ versus $6 \%$ ( $\mathrm{p}<0.00001)$. Adverse events leading to withdrawal from the study occurred in $8 \%$ of cases on Sunitinib and $13 \%$ on IF. Data are not yet mature for the assessment of overall survival, but the investigators concluded that Sunitinib should be considered the new standard for the first line treatment of metastatic RCC. Sunitinib was also evaluated in patients who have failed prior Bevacizumab therapy in a phase II trial (61 cases). An ORR of $16 \%$ was demonstrated with $61 \%$ of cases with stable disease.

\section{Sorafenib}

In December 2005, the FDA approved Sorafenib for the treatment of patients with advanced RCC [47]. The approval was based on randomized trials evaluating Sorafenib, in particular in cases in progression after cytokine therapies. Sorafenib has been evaluated as monotherapy in four phase I trials with distinct schedules of administration: interrupted dosing and continuous administration [48]. In these trials 173 patients with advanced solid tumors were enclosed. Sorafenib was generally well tolerated at dosing $\leq 400 \mathrm{mg}$ bid. The most frequently reported adverse events related to the drug were fatigue(40\%), anorexia (35\%), diarrhoea (34\%) and rush hand-foot skin reaction $(25 \%)$. These adverse events were mainly <grade $3(90 \%)$ and were resolved either reducing the dose or discontinuing Sorafenib. A treatment related hypertension was observed in $5-11 \%$ of cases. The maximal tolerated dose for Sorafenib was defined in these trials as $400 \mathrm{mg}$ bid. The hypothesis is that reactions are related to EGFR and Raf inhibition, whereas hypertension to VEGF inhibition [49].

A first phase II randomized discontinuation trial evaluated the effect of Sorafenib in 202 patients with advanced RCC (all with ECOG score 0 or $1 ; 75 \%$ clear RCC; $89 \%$ with prior nephrectomy; $76 \%$ with prior IL-2 or IF therapy) [50].

Patients received Sorafenib $400 \mathrm{mg}$ bid during an initial run-in period and thereafter (12 weeks) cases with changes in tumor measurement $<25 \%$ were randomized to Sorafenib versus placebo for other 12 weeks. Patients with $25 \%$ tumor growth discontinued treatment whereas patients with $25 \%$ tumor shrinkage continued open-label Sorafenib. At 24 weeks, $50 \%$ of Sorafenib treated patients were progression-free versus $18 \%$ of the placebo group $(p=0.007)$. Median PFS was 24 weeks in Sorafenib versus 6 weeks 
in placebo group $(\mathrm{p}=0.008)$. A randomized phase II trial of Sorafenib versus IF in untreated metastatic RCC is ongoing to define the role of this drug in this setting [38]. The clinical advantage of Sorafenib was than confirmed in a multicenter double blind, randomized phase III trial of Sorafenib versus placebo [51]. In this trial 905 cases with advanced RCC with clear cell histology, failure of prior systemic cytokine therapy, ECOG score 0-1, almost submitted to nephrectomy, were included. In this trial, $2 \%$ of patients receiving the drug had an objective response (RECIST criteria), and stable disease was found in $78 \%$ of cases ( $55 \%$ in placebo arm). The median PFS was 24 weeks in the Sorafenib group an 12 weeks in the placebo group ( $\mathrm{p}=0.0001)$. Based on these results the study was modified to allow cross-over from placebo to Sorafenib. Although results are not mature for a overall survival evaluation, a trend toward improved survival for the Sorafenib treated patients $(\mathrm{HR}=0.72 ; \mathrm{p}=0.018)$ was found $[49,50]$.

\section{Combination Therapies Using Sorafenib or Sunitinib}

The rationale for targeting VEGF in advanced RCC is important, but also other factors, including PDGF, are able to influence the angiogenenetic process in this tumor. Therefore, enhanced antitumors activity, could be obtained by a simultaneous targeting of different factors involved in this process. Different preclinical studies suggest that IF has anti-angiogenic properties [38] and that combining 2 or more agents with anti-angiogenic properties may result in additive or synergistic anti-tumor activity.

Clinical trials have been started to examine the possibility of combination targeted therapies in advanced RCC. Combination of Bevacizumab and IF versus IF monothrerapy has been evaluated in two randomized phase III trials as first line therapy in metastatic RCC [52]. However these trials did not fully define the additive or synergic effects of this combination regimen due to the absence of a Bevacizumab monotherapy control arm. Two phase II trials combining sorafenib $400 \mathrm{mg}$ bid with IF 3-times week have been reported $[53,54]$. These trials suggest that this combination regimen can be safely used in untreated advanced RCC patients. Among 24 cases valuable for response, the ORR by RECIST criteria was $42 \%$ (38\% partial and $4 \%$ complete response) ; moreover $46 \%$ of cases showed stable disease. In a phase I trial, 24 patients with metastatic RCC received Bevacizumab plus Sorafenib. The combination showed preliminary evidence of anti-tumor activity but a doselimiting toxicity related to hand-foot syndrome, anorexia, and fatigue, was reported [55]. Combining of anti VEGF strategies with agents targeting other relevant mechanisms in advanced RCC have been also planned. A phase II trial [40] analyzed the combination of Bevacizumab with Erlotinib (EGFR inhibitor). The trial showed that Erlotinib does not significantly add to the clinical efficacy of Bevacizumab. A promising combination strategy considered antiVEGF drugs with Temsirolimus, a mammalian target of rapamycin inhibitor (PFS of 3.7 months versus 1.9 months, $p=0.0001$ in a phase III trial comparing Temsirolimus with IF in advanced RCC) [56].

\section{FUTURE POINTS TO BE ADDRESSED}

There are at present no precise guidelines on how to treat advanced RCC as first line treatment or at failure as second line treatment, using targeted agents. The information that we presented support the clinical rationale for targeting angiogenesis and the VEGF pathways and they show that this strategy is feasible and it can result in tumor shrinkage and PFS advantages. To better use this kind of targeted therapy, different points should be developed in the future: 1) the identification of the clinical characteristics of RCC patients that can be used to predict outcomes and responses to therapy; 2) the definition of the differences among different compounds; 3) the advantage of combination therapies; 4) the possibility of sequential treatments.

\section{Patient Selection}

The pre-selection of patients most likely to respond to targeted therapies and anti-VEGF therapy, should be crucial to optimize these agents in the clinical setting. Advanced RCC, prior nephrectomy, a clear cell histology were inclusion criteria in almost trials. Clear cell histology criterion was based on the biology of VHL inactivation and subsequent VEGF hyper-expression, which is confirmed to this histologic type of RCC [38]. In some trials, however, there have been reports of activity of anti-VEGF agents also in cases with non clear cell histology [38]. This point should be better defined in future trials.

The median PFS of 13 months obtained with VEGF targeted agents in metastatic RCC is significantly superior to that reported in other trials involving earlier therapies [57]. The real value of PFS, however, may change in relation of the selected group of patients who is considered for the treatment. Predictive biomarkers of response should also be largely investigated and VEGF expression is an obvious candidate.

Another important point is the statistically similar PFS of patients receiving VEGF targeted therapy for metastatic disease as first line or second line treatment [57]. This finding supports the hypothesis that the biology of RCC that mediates response to anti VEGF therapies may not be influenced by prior exposure to cytokine therapies. This represent another important point that should be better defined.

\section{Differences Among Different Compounds}

The recent approval of 2 mult-targeted TK inhibitors that both modify the natural history of RCC (Sorafenib and Sunitinib) must induce to compare these two drugs together. Also this approach helps to determine how best to use them in metastatic RCC. Differences have been analyzed graphically in the form of a dendogram [58]. In this kind of analysis, Sunitinib bound 73 kinases in addition to VEGFR, whereas Sorafenib bound 40 additional kinases. The clinical relevance of inhibiting " off target " kinases hs not been yet defined in terms of clinical advantage. Sorafenib, unlike Sunitinib, is also an inhibitor of Raf kinase. Also in this case, the relevance of EGFR and Raf system in RCC is controversial and therefore it remains uncertain whether Raf activity inhibition has a clinical role in the treatment of advanced RCC. It should be interesting to define a possible different role of Sorafenib and Sunitinib as first line therapy or after cytokine treatment resistance. The two drugs should also be extensively examined and compared in the treatment of RCC patients with non clear tumors. In the phase III trials with Sorafenib and Sunitinib, non clear cell histologies were specifically excluded. It is possible that one drug over the other may have a potential role in the treatment of papillary and chromophobe RCC.

Moreover, considering that Sorafenib can target endothelial cells also through Raf pathways rather than through VEGFR pathways, it could be possible that Sorafenib may overcome a resistance of the tumor to VEGF inhibitors such as Sunitinib [38].

To complete the comparison of the two drugs Sorafenib and Sunitinib, differences must be considered in terms of toxicity (lower grade 3 toxicity, rate with Sorafenib), response rate (higher objective response rate with Sunitinib) and administration (continuous with Sorafenib and intermittent with Sunitinib) [38].

All these suggestions strongly support the need for a direct comparison of Sorafenib and Sunitinib. An Eastern Cooperative Oncology Group (ECOG) phase III trial, with patients randomized to Sorafenib versus Sunitinib versus placebo is ongoing [38].

\section{Advantages of Combination Therapy and Role of Sequential Treatments}

To understand the role and the advantage of a combination therapy in this setting, different questions should be addressed: 1) 
which target is most important? 2) which agent better inhibits each target? 3) Is there a synergic effect using combination therapy? 4) Which is the advantage to be reached (improvement in the rate and duration of clinical response, prevention of clinical resistance)? 5) Which population may better respond (first line therapy, cytokine resistant tumors, second or third line targeted therapy)?

Kaekin et al. [59] underlined two different concepts of combination targeted therapy for RCC. "Horizontal blockade" is when different target molecules are at the same time and individually inhibited: tumor cells (EGFR), endothelial cells (VEGFR) and pericyte (PDGF). To obtain this kind of blockade, specific single inhibitors or multi-targeted agents (Sorafenib and Sunitinib) could be used. "Vertical blockade" is when a specific pathway is targeted and than inhibited at 2 or more different levels (for example VEGF and TK).

In RCC the horizontal blockade is intended to block tumor progression acting at different levels, either directly on tumor cells or indirectly on angiogenesis. The vertical blockade helps to resolve the problem of resistance that may develop during targeted therapy. In terms of resistance to targeted therapy for advanced RCC, there are no guidelines at present on how sequencing drugs or how to treat RCC after failure of first or second line agents. The hypothesis of no cross-reactivity among the different targeted therapies has set the basis for a strategy based on a sequential therapy [26]. The determination of the correct sequence in using targeted therapy in RCC is a very important point that also support the rationale for continuing targeting the angiogenic pathways throughout progression and resistance of the RCC tumor.

\section{FINAL COMMENT}

The large number of scientific articles published in the 2007 literature (25 publications on Pubmed only from January to July 2007) on the role of targeted therapy (in particular anti-angiogenic / anti-VEGF) in advanced RCC, strongly underlines the actual relevance and the innovative role of this therapeutic approach in a setting in which other previous systemic treatments (chemotherapy, radiotherapy and partially also cytokine inhibitors) failed.

Targeted therapies with Sorafenib and Sunitinb have been approved by FDA and now are revolutioning how we clinically approach advanced RCC. These new therapeutic approaches sustain the role of surgery, either in terms of cytoreductive nephrectomy or metastatectomy, as first treatment for advanced RCC. On the contrary the activity of these agents without prior nephrectomy remains undefined.

It is possible to predict a future extension for the indications of these targeted therapies in RCC, for example as adjuvant to nephrectomy in high risk non metastatic cases.

We must also underline limits of these therapies. The novel agents are more likely to be cytostatic than cytotoxic and therefore we still fall to achieve complete and durable responses, but only advantages in terms of PFS or as disease stabilization can be expected.

A multidisciplinary approach involving both urologists and oncologists is required when treating advanced RCC cases, and no single therapy should be considered as standard of care.

In this scenarios, patients should be informed regarding the utility of surgery, advantages and risks of each novel targeted approach, finality and possibility of these therapies, so to individualize the treatment for each single RCC case.

\section{REFERENCES}

[1] Jemal A, Siegel R, Ward E, et al:: Cancer statistics, 2006. Cancer J. Clin.2006; 56:106-130.

[2] Jemal A, Siegel R, Ward E, Murray T, Xu J, Thun MJ.: Cancer statistics, 2007. Cancer J. Clin. 2007; 57:43-66.
Zisman A, Pantuck AJ, Wieder J, et al.: Risk group assessment and clinical outcome algorithm to predict the natural history of patients with surgically resected renal cell carcinoma. J Clin. Oncol. 2002; 20:4559-4566.

Bukowski RM: Natural history and therapy of metastatic renal cell carcinoma: the role of interleukin-2. Cancer 1997; 80:1198-1200.

Fyfe G, Fisher RI, Rosenberg SA, Snzol M, Parkinson DR, Louie AC: Results of treatment of 255 patients with metastatic renal cell carcinoma who received high-dose recombinant interlukin-2 therapy. J. Clin. Oncol. 1995; 13: 688-696.

No author listed: Interferon-alpha and survival in metastatic renal cell carcinoma: early results of a randomized controlled trial. Medical research council renal cancer collaborators. Lancet 1999; 353:14-17.

Mcdermott DF, Regan MN, Clark JI, et al.: Randomized phase III trial of high-dose interleukin-2 versus subcutaneous interleukine-2 and interferon in patients with metastatic renal cell carcinoma. J. Clin. Oncol. 2005; 23:133141.

Ferrara N. Vascular endothelial growth factor as a target for anticancer therapy. The Oncologist 2004; 9:2-10. tion targeted therapy in renal cell carcer. Clin. Cancer Res. 2007; 13:764769.

Pantuck AJ, Zeng G, Belldegrun AS, Figlin RA. Pathobiology, prognosis, and targeted therapy for renal cell carcinoma: exploiting the hypoxia induced pathway. Clin. Cancer Res. 2003; 9: 4641-4652.

Klatte T, Pantuck AJ, Kleid MD, Belldegrun AS. Understanding the natural biology of kidney cancer: implications for targeted cancer therapy. Rev. Urol. 2007; 9: 47-56.

Gnarra JR, Zhou S, Merrill MJ, et al. Post-transcriptional regulation of vascular endothelial growth factor mRNA by the product of the VHL tumor suppressor gene. Proc. Natl. Acad. Sci. USA 1996; 93:10589-94.

Turner KJ, Moore JW, Jones A, et al. expression of hypoxia-inducible factors in human renal cancer: relationship to angiogenesis and to the Von Hippel Lindau gene mutation. Cancer Res. 2002; 62:2957-61.

Gabrilovich DI, Ishida T, Nadaf S, Ohm JE, Carbone DP. Antibodies to vascular endothelial growth factor enhance the efficacy of cancer immunotherapy by improving endogenous dendritic cell function. Clin. Cancer Res. 1999; 5: 2963-70.

Jacobsen J, Grankvist K, Rasmuson T, Bergh A, Landberg G, Ljungberg B. Expression of vascular endothelial growth factor protein in human renal cell carcinoma. BJU Int 2004; 93: 297-302.

Shibuya M, Claesson-Welsh L. Signal transduction by VEGF receptors in regulation of angiogenesis and liphangiogenesis. Exp. Cell Res. 2006; 312 549-560.

Byrne AM, Bouchier-Hayes DJ, Harmey JH. Angiogenic and cell survival functions of vascular endothelial growth factor (VEGF). J. Cell Mol. Med. 2005; 9: 777-794.

Leppert JT, Lam JS, Yu H, et al. Targeting the vascular endothelial growth factor pathway in renal cell carcinoma. J. Clin. Oncol. 2005; 23:4536.

Lam JS, Leppert JT, Yu H, et al. Expression of the vascular endothelial growth factor family in tumor dissemination and disease free survival in clear cell renal cell carcinoma. J. Clin. Oncol. 2005; 23:4538.

Rioux-Leclerq N, Fergelot P, Zerrouki S, et al. Plasma level and tissue expression of vascular endothelial growth factor in renal cell carcinoma: a prospective study of 50 cases. Human Pathology 2007 (Epub ahead of print). Bornes S, Boulard M, Hieblot C, et al. Control of the vascular endothelial growth factor internal ribosome entry site (IRES) activity and translation initiation by alternatively spliced coding sequences. J. Biol. Chem. 2004; 279: 18717-26.

Jacobsen J, Rasmuson T, Grankvist K, Ljungberg B. Vascular endothelial growth factor as prognostic factor in renal cell carcinoma. J. Urol. 2000; 163 343-7.

Edgren M, Lennernas B, Larson A, Kalkner KM. Angiogenic factors: vascular endothelial growth factor VEGF and basic fibroblast growth factor b-FGF are not necessarily elevated in patients with advanced renal cell carcinoma. Anticancer Res. 2001; 21: 1423-30.

Chow L and Eckhardt G. Sunitinib:from rational design to clinical efficacy. J. Clin. Oncol. 2007; 7:884-96.

Pawson T: Regulation and targets of receptor tyrosine kinases. Eur. J. Cancer 2002; 38:s3-s10.

Bellmunt J, Montagut C,Albiol S, et al. Present strategies in the treatment of metastatic renal cell carcinoma: an update on molecular targeting agents. BJU Int 2007; 99:274-80.

Yang XD, Jia Xc,Corvalan JR, Wang P, Davis CG. Development of ABXEGF, a fully human anti-EGF receptor monoclonal antibody, for cancer therapy. Crit. Rev. Oncol. Hematol. 2001; 38:17-23.

Motzer RJ, Amato R, Todd M, et al. Phase II trial of antiepidermal growth factor receptor antibody $\mathrm{C} 225$ in patients with advanced renal cell carcinoma. Invest New Drugs 2003; 21: 99-101.

Figlin RA, Seligson DB, Wu H, et al. Characterization of the mTOR pathway in renal cell carcinoma and its use in predicting patient selection for agents targeting this pathway. J. Clin. Oncol. 2005; 23: 4539.

Schmidt L, Junker K, Nakaigawa G, et al. Novel mutations of the MET proto-oncogene in papillary renal carcinomas. Oncogene 1999; 18: 2343-50. Leppert JT, Lam JS, Pantuck AJ, Figlin RA, Belldegrun AS et al. Carbonic anhydrase IX and the future of molecular markers in renal cell carcinoma. 
BJU Int 2005; 96: 281-85.

[32] Wood CG. The role of cytoreductive nephrectomy in the management of metastatic renal cell carcinoma. Urol. Clin. North Am. 2003, 30: 581-88.

[33] Flanigan RC, Salmon SE, Blumenstein BA, et al. Nephrectomy followed by interferon alfa-2b compared with interferon alfa-2b alone for metastatic renal cell cancer. N. Engl. J. Med. 2001; 345: 1655-59.

[34] Mickisch GH, Garin A, van Poppel H, et al. Radical nephrectomy plus interferon-alfa-based immunotherapy compared with interferon alfa alone in metastatic renal cell carcinoma: a randomized trial. Lancet 2001; 358: 96670.

[35] Garcia JA, Rini BI. Recent progress in the management of advanced renal cell carcinoma. Cancer J. Clin. 2007; 57: 112-125.

[36] Frank I, Blute ML, Cheville JC Lohse CM, Weaver AL, Zincke H. An outcome prediction model for patients with clear renal cell carcinoma treated with radical nephrectomy based on tumor stage, size, grade and necrosis: theSSIGN score. J. Urol. 2002; 168: 2395-2400.

[37] Presta LG, Chen $\mathrm{H}$, O'Connor SJ, et al. Humanization of anti-vascular endothelial growth factor monoclonal antibody for the therapy of solid tumors and other disorders. Cancer Res. 1997; 57: 4593-99.

[38] Rini BI Vascular endothelial growth factor taregeted therapy in renal cell carcinoma: current status and future directions. Clin. Cancer Res. 2007; 13: 1098-1106.

[39] Yang JC, Hawoth L, Sherry RM, et al. A randomized trial with Bevacizumab, an anti-vascular endothelial growth factor antibody, for metastatic renal cancer. N. Engl. J Med. 2003; 349: 427-434.

[40] Bukowsy RM, Kabbinavar F, Figlin RA, et al. Bevacizumab with or without Erlotinib in metastatic renal cell carcinoma (RCC). J. Clin. Oncol. 2006; 24: 222s.

[41] Sonpavde G, Hutson TE. Recent advances in the therapy of renal cancer. Expert Opin. Biol. Ther. 2007; 7: 233-242.

[42] Faivre S, Delbaldo C, Vera K, et al. Safety, pharmacokinetic, an antitumor activity of SU11248, a novel oral multitarget tyrosine kinase inhibitor, in patients with cancer. J. Clin. Oncol. 2006; 24: 25-35.

[43] Houk B, Bello C, Garret M, et al. Population pharmacodynamic metaanalysis of SU11248 efficacy and tolerability endpoints in gastrointestinal stromal tumors (GIST), metastatic renal cell carcinoma (MRCC) and solid tumor patients. Presented at EORTC/AACR/NCI Molecular Target Meeting, Philadelphia, PA, November 14-18, 2005.

[44] Motzer RJ, Michaelson MD, Redman BG, et al. Activity of SU11248, a multitargeted inhibitor of vascular endothelial growth factor receptor and platelet-derived growth factor receptor, in patients with metastatic renal cell carcinoma. J. Clin. Oncol. 2006; 24: 16-24.

[45] Motzer RJ, Rini BI, Bukowsky RM, et al. Sunitinib in patients with metas- tatic renal cell carcinoma. JAMA 2006; 295: 2516-2524.

[46] Motzer RJ, Hutson TE, Tomczak P, et al. Sunitinib versus interferon alfa in metastatic renal cell carcinoma. N. Engl. J. Med. 2007; 356: 115-124.

[47] Goodman VL, Rock EP, Dagher R, et al. Sunitinib for the treatment of Imatinib refractory or intolerant gastrointestinal stromal tumors and advanced renal cell carcinoma. Clin. Cancer Res. 2007; 13: 1367-1373.

[48] Strumberg D, Clark JW. Awada A, et al. Safety, pharmacokinetics and preliminary antitumor activity of sorafenib: a review of four phase I trials in patients with advanced refractory solid tumors. The Oncologist 2007; 12 : 426-437.

[49] Flaherty KT, Sorafenib: delivering a targeted drug to the right targets. Expert Rev. Anticancer Ther. 2007; 7: 617-626.

[50] Ratain MJ, Eisen t, Stadler WM, et al. Phase II placebo-controlled randomized discontinuation trial of sorafenib in patients with metastatic renal cel carcinoma. J. Clin. Oncol. 2006; 24: 2505-2512.

[51] Escudier B, Eisen T, Stadler WM, et al. Sorafenib in advanced clear cell renal cell carcinoma. N. Engl. J. Med. 2007; 356: 125-34

[52] Rini BI, Halabi S, Taylor J, Small EJ, Scilsky RL, Cancel and Leukemia group B 90206: a randomized phase III trial of interferon-a or interferon-a plus antivascular endothelial growth factor antibody (Bevacizumab) in metastatic renal cell carcinoma. Clin. Cancer Res. 2004; 10: 2584-6.

[53] Gollob JA, Rathmell WK, Richmond TM, et al. Phase II trial of Sorafenib plus interferon-alpha $2 \mathrm{~b}$ as first or second line therapy in patients with metastatic renal cell cancer. J. Clin. Oncol. 2007; 25: 3288-95.

[54] Ryan CW, Goldman BH, Lara PN, et al. Sorafenib plus interferon alpha $2 \mathrm{~b}$ as first line therapy for advanced renal cell carcinoma: SWOG 0412. J. Clin Oncol. 2007; 25: 3296-301.

[55] Sosman JA, Flaherty K, Atkins MB, et al. Phase I/II trial of Sorafenib with Bevacizumab in metastatic renal cell cancer patients. J. Clin. Oncol. 2006; 24: $128 \mathrm{~s}$.

[56] Hudes G, Carducci M, Tomczak P, et al. a phase III randomized £-arm study of Temsirolimus (TEMSR) or interferon-a or in the combination of TEMSR+INF in the treatment of first line, poor risk patients with advanced renal cell carcinoma. J. Clin. Oncol. 2006; 24: 2s.

[57] Choueiri TK, Garcia JA, Elson P, et al. Clinical factors associate with outcome in patients with metastatic clear-cell renal cell carcinoma treated with vascular endothelial growth factor targeted therapy. Cancer 2007; 110: 54350.

[58] Stein MN, Flaherty KT, Sorafenib and Sunitinib in renal cell carcinoma. Clin. Cancer Res. 2007; 13: 3765-3770

[59] Kaelin WG, The von Hippel Lindau gene, kidney cancer and oxygen sensing. J. AM. Soc. Nephrol. 2003; 14: 2703-11. 